\title{
El consentimiento informado en clínica: inquietudes persistentes
}

\author{
MIGUEL KOTTOW
}

\section{Informed consent in clinical practice: persistent doubts}

Informed consent is the core aspect of the patient-physician relationship. Since its beginnings, clinical bioethics was opposed to the authoritarian paternalism characteristic of medicine since the $19^{\text {th }}$ century. The informed consent was developed to provide patients with sufficient information to allow autonomous decisions when faced with medical diagnostic and therapeutic alternatives. In spite of bioethics' effort to perfect informed consent, the discipline has been unable to avoid informed consent from becoming an impersonal and administrative procedure. Even though the major goal of this procedure is to provide sufficient information to allow patients an objective weighting of benefits and risks of medical practice, the uncertainties of medicine make full disclosure unattainable. Collecting more information finally leads to indecision and ultimate trust in medical advice. The clinical encounter is fundamentally a fiduciary relationship, and bioethics ought to accept that its main objective is to strengthen the trust bond that is essential to the clinical encounter. This goal may become incompatible with the quest for unlimited autonomy. Patients often will only require information as long as they distrust that medical institutions and their professionals are considering their interests and needs. The main proposal of this article is to temper bioethics' insistence on autonomy, and accept that patients essentially seek to be protected and cared for. Informed consent ought to relent its efforts at full autonomy to the benefit of trustworthiness in medicine, and trust in clinical practice.

(Rev Med Chile 2016; 144: 1459-1463)

Key words: Bioethical issues; Consent Forms; Informed Consent; Personal Autonomy; Trust.

$\mathrm{E}$ desarrollo del CI se enfocó desde los comienzos de la bioética en eliminar el paternalismo médico y reemplazarlo por la autonomía decisional del paciente, cultivada por un proceso acabado de consentimiento voluntario e informado. El tema fue central cuando la bioética ponía el máximo énfasis en la relación médico/ paciente y en el encuentro clínico que debía sustentarse en la información entregada por el terapeuta y en la voluntad del paciente por decidir entre las diversas sugerencias de acción u omisión. Así lo entendió la Declaración de Lisboa (1981/1995): "El paciente tiene derecho a la autodeterminación
Facultad de Ciencias de la Salud, Universidad Central de Chile. Santiago, Chile.

Recibido el 29 de junio de 2016, aceptado el 11 de octubre de 2016.

Corrrespondencia a: Miguel Kottow, MD, MA. Facultad de Ciencias de la Salud, Universidad Central de Chile. J. Miguel de la Barra 521,

Depto. 4-B

Miguel.kottow@ucentral.cl y a tomar decisiones libremente en relación a su persona". Pese a todos los problemas que esta formulación produce en la práctica médica, la claridad conceptual del CI parecía asegurada, justificando la afirmación que "[L]a noción de consentimiento informado se encuentra actualmente en el centro de la medicina"1. En el prefacio del texto más completo sobre la materia, se señala que "nuestra meta primaria al escribir este libro ha sido proveer una respuesta satisfactoria a la pregunta " $¿ Q u e ́$ es el consentimiento informado?"2. Estas intenciones no obstante, el CI continúa siendo tema de controversias académicas y de incomodidad práctica ${ }^{3}$. 
Las múltiples y cotidianas distorsiones que el CI adolece en la práctica clínica, han terminado por transformar el proceso comunicativo interpersonal en un procedimiento burocrático y muchas veces secretarial. Aun cuando se depurase el CI al máximo, sería una herramienta cuya fundamentación teórica y objetivos prácticos requieren ser reflexionados y reformulados.

\section{Desde la teoría}

El concepto de autonomía proviene de Kant y su convicción de que el rasgo antropológico esencial del ser humano es su voluntad libre, independiente de toda contingencia o condición. El deber moral consiste en ser fiel a la buena voluntad que se rige por el imperativo categórico de tratar a toda persona como fin y no sólo como medio. Lo propuesto por el racionalismo kantiano es insostenible en la práctica siempre condicionada por contextos y situaciones sociales, el reconocimiento y respeto de las personas interactuantes. La hegemonía otorgada a la autonomía por el principialismo de Georgetown (autonomía, beneficencia, no maleficencia, justicia), y su presencia en la tétrada principialista europea (vulnerabilidad, dignidad, integridad, autonomía), llevan a entender la autonomía no como un deber kantiano, sino como un derecho: "En una sociedad pluralista moderna, la autonomía personal, el derecho de elegir el propio modo de vida, es considerado el valor supremo" ". Entendida como un derecho, la autonomía hace lo contrario de lo aceptable para Kant al convertirse en instrumento de logros a alcanzar con el refinamiento máximo del CI en medio de numerosas condicionantes contextuales. El carácter utilitarista del CI lo somete a criterios de factibilidad, eficacia, relevancia, veracidad y prudencia, cultivados en el diálogo y evitando que el proceso se marchite en una mecanización burocrática.

\section{Consideraciones jurídicas}

La incorporación del CI en el seno de la práctica médica se refuerza con el desarrollo de una nutrida jurisprudencia afirmativa del derecho a decisión informada, y a la penalización de la práctica médica si produce daños que no hubiesen sido presentados al paciente como posible riesgos. El médico que interviene sin el consentimiento del paciente, comete una agresión corporal, siendo responsable por daños resultantes., La intervención sin expreso consentimiento adecuadamente informado es motivo de querella cuando el resultado es indeseado por el paciente $e^{5,6}$. El CI plantea esencialmente un reto ético ${ }^{2}$, mas igualmente se entiende que este fundamento ético debe ser plasmado en leyes que condicionen, regulen y limiten un derecho de autonomía que ha de respetar y convivir con otros derechos y deberes -del médico, del sistema de salud, de valores culturales, de tradiciones sociales-.

La relevancia ética del CI en la práctica médico-clínica requiere un soporte jurídico, en Chile plasmado en la regulación legal de derechos y deberes de los pacientes (2012). En relación a autonomía y CI, expertos en derecho comentan que esta ley "más que respuestas viene a plantear un sinnúmero de nuevas interrogantes" a sugerir que "es posible pensar que la nueva ley limita severamente la autonomía de los pacientes sobre bases que puedan estar equivocadas $\mathrm{o}$, al menos, ser objetables"8.

\section{Consentimiento informado y paternalismo}

La oposición al paternalismo se refiere a la forma autoritaria que niega al paciente competente el ejercicio de autonomía, a diferenciar del paternalismo protector que asume decisiones médicas para compensar lagunas o ausencias del discernimiento autónomo del paciente. Un aspecto adicional a considerar es que el ejercicio de autonomía es dependiente del contexto social. La enfermedad no necesariamente reduce el discernimiento, pero sí presenta una brecha de información que es mayor en sociedades desiguales, donde los desposeídos viven en desventaja cognitiva que el médico ha de respetar y reducir, pero sin tensionar la decisión autónoma más allá de lo que el paciente desee.

Los primeros códigos de ética médica (Gregory, Beddoes, Percival) enfatizaron que la profesión médica debía ganarse la confianza pública en su eficacia clínica y prestancia ética. El énfasis en el CI como forma de robustecer la autonomía del paciente es en gran medida causado por la pérdida de confianza en instituciones y profesiones. La desconfianza se acrecienta a todo nivel, llevando 
al ciudadano a cuestionar la entereza técnica y ética de los servicios que solicita. A medida que se profundiza y complejiza el nivel de información, aparecen las incertidumbres de la medicina que confunden más que esclarecen al paciente. Para la práctica médica, el afán informativo va en directa relación con la desconfianza en la atención médica sobre todo cuando es instrumentalmente sofisticada, los beneficios terapéuticos son más inciertos y los efectos indeseados más probables. Se produce un equilibrio sociológico inestable entre admiración por el progreso tecnocientífico y la desconfianza en los servicios que provee ${ }^{9,10}$.

\section{Inestabilidad conceptual del consentimiento informado}

A pesar de una teorización insuficiente y una judicialización ambigua, el CI mantiene su centralidad en la interacción autónoma de pacientes y profesionales encargados de proveer atención médica. A lo largo de varios decenios de disquisiciones bioéticas, el tema continúa siendo discutido, sin formular recomendaciones claras para que la decisión informada del paciente se desarrolle en forma satisfactoria para todos. El debate teórico se ha extraviado por concentrarse en situaciones excepcionales, antes que depurar los aspectos relevantes en el encuentro clínico cotidiano, prefiriendo desmenuzar la legitimidad del $\mathrm{CI}$ en personas con enfermedades irrecuperables y letales a corto plazo, la autonomía de pacientes en estados inciertos de competencia mental, la legitimidad de decisiones vicariantes o extemporáneas -testamentos en vida-, el ejercicio de autonomía cuando ella implica y potencialmente lesiona a terceros. No obstante, las situaciones inusuales no permiten extrapolar desde la excepción, ni sentar precedentes éticos o jurídicos que sean generalizables, como lo ha pretendido lograr, entre otros, el casuismo ${ }^{11}$.

Por otra parte, la bioética se ha empecinado en suponer que, mientras más se detalle y profundice el proceso de CI hasta depurarlo de todo resto de paternalismo, mayor será la autonomía del paciente ${ }^{12}$. El CI es presentado no sólo como necesario sino también como suficiente para el resguardo ético del paciente. Si el CI no logra este desiderátum es, según sus defensores, por las fallas procedimentales que lo aquejan y que, en principio, serían removibles. Dadas estas limitaciones, se ha propuesto apoyar el proceso cognitivo con "especialistas en consentimiento informado" que asistan a los pacientes en entender información, estabilizar sus reacciones emocionales y ponderar más objetivamente las opciones médicas sugeridas $^{13}$. Por el contrario, se argumenta que el paciente no quiere, ni se siente en condiciones, de ejercer autonomía cabal sobre sus decisiones médicas, en tanto el médico considera, de buena $\mathrm{fe}$, que la prestancia clínica requiere un cierto grado de autoridad por su parte, y una dosis de disciplina del enfermo mostrada en una adecuada adherencia a tratamientos acordados. Todo apunta a la necesidad impostergable de recuperar una relación participativa, de confianza, libre de autoritarismos, con narrativa e información relevantes.

El ejercicio de máxima autonomía sustentada por el CI tiene deficiencias intrínsecas, pues la situación clínica se matiza con situaciones contextuales, biográficas, de emocionalidad que dificultan y aun desaconsejan el ejercicio de autonomía racional pura, haciendo del CI un proceso inherentemente opaco ${ }^{14,15}$.

El énfasis irrestricto en la autonomía, como ejercida por la bioética anglosajona ${ }^{16}$, puede llevar a extravagancias teóricas, como es la propuesta de que la reconocida autonomía del paciente por rehusar tratamientos debiera fortalecer la automedicación y la independencia para acceder a medicamentos sin prescripción médica ${ }^{17}$. Complica aún más el tema la propuesta de que el CI permita descargar la responsabilidad médica en el paciente, que asume y acepta los riesgos posibles por cuanto ha sido informado de ellos ${ }^{18}$.

\section{El encuentro clínico fiduciario}

La insistencia en un proceso acabado de CI que sea "oportuno, adecuado, suficiente y comprensible" compartidos estos adjetivos. La práctica médica se encuentra frecuentemente con el cansancio informativo del paciente o su explícita negativa a recibir información ${ }^{19}$, apoyándose, más pronto que tarde, en la recomendación médica, ya que solicita ayuda terapéutica y no la exposición neutra de alternativas entre las cuales ha de decidir en forma autónoma. Los clásicos de la bioética han insistido en el carácter sodalicio (Laín Entralgo) y fiduciario (Ramsey, Pellegrino) de la relación entre paciente y médico ${ }^{20}$. El CI ha de ser veraz y 
atingente a los valores del paciente; más que una empecinada y detallada completitud, lo central siendo un esclarecimiento relevante, libre de tecnicismos, y la disposición a ofrecer más información si el paciente así lo solicita. La experiencia médica confirma que existe una relación inversa entre confianza y requerimientos de información detallada: el paciente que confía en la competencia y honestidad de su médico, limita su indagación a conocer los riesgos y beneficios de las diversas alternativas $y$, por lo general, acepta lo médicamente más aconsejable ${ }^{21}$. El paciente acude por propia voluntad en busca de consejo y asesoría, y no solamente a solicitar información y competencia técnica.

Para una bioética más acorde con la realidad social y cultural de los países latinoamericanos, el CI se engarza en una relación médico-paciente más relacional que contractual, con mayor énfasis en protección. Tema a tratar con cautela, puesto que la distinción entre una perspectiva anglosajona y una bioética latinoamericana sigue sometida a debate, algunos prefiriendo hablar de bioética en Latinoamérica ${ }^{22}$.

Un objetivo más cercano a la enseñanza y práctica médica ${ }^{23}$, sindica al CI como un instrumento que enfatiza la relación fiduciaria entre pacientes y médicos, amenazada por el clima contemporáneo de desconfianza y la experiencia de verdaderos abusos, produciendo "una caída en la confianza del profesionalismo y del juicio profesional" 24 . La restitución de la confianza requiere, primariamente, la cabalidad ética del proceso mismo del CI, veraz y relevante más que técnicamente exhaustivo, actuando como instrumento de fortalecimiento del vínculo fiduciario entre paciente y terapeuta. También aquí se cae fácilmente en exageraciones teóricas, al tratar de recuperar tanta confianza que haría superflua la información y la debida participación decisional del paciente ${ }^{25}$.

\section{Conclusión}

La ciudadanía ve confirmada su desconfianza en instituciones y prácticas sociales como la política, la economía, específicamente para el presente contexto, en la práctica médica ${ }^{26}$. Críticos de la medicina hablan de "deshumanización", cuando en rigor debieran referirse a "despersonalización" La tendencia al positivismo, a la medicina basada en evidencia, el predominio de la medicina administrada y de la expedición contractual por sobre el compromiso fiduciario, llevan a enfrentar al paciente cada vez más con un sistema médico que reduce y tecnifica el encuentro clínico interpersonal. La narrativa del paciente es desatendida, la semiología del cuerpo es reemplazada por la exploración técnica, las explicaciones médicas son parcas, el factor tiempo reduce la duración del encuentro clínico, llevando a una medicina despersonalizada. Las dificultades de acceso, las limitaciones a la libre elección del médico, el frecuente ingreso a través de servicios de urgencia, aumentan las inseguridades y desconfianzas del enfermo.

El largamente sostenido afán de eliminar mediante el CI todo vestigio de paternalismo y robustecer al máximo la autonomía del paciente, está creando más problemas que soluciones en la ética clínica. Habrá de restituirse la mutua confianza entre paciente y médico mediante una construcción cotidiana y permanente, el médico recuperando confiabilidad al ejercer criterio diagnóstico y terapéutico orientado a las necesidades e intereses del paciente ${ }^{27}$. Este a su vez ha de ser confiable en su adhesión a los esquemas de control, cumplimiento de indicaciones terapéuticas y participación en lo que plausiblemente le ha sugerido el médico. Este último punto cobra cada vez mayor importancia a medida que el paciente es requerido de participar activamente en promover su salud y cuidar sus enfermedades, especialmente las crónicas e inestables ${ }^{28}$.

La relación sodalicia y de mutua confianza sigue siendo el fundamento de la práctica médica, aún cuando severamente tensionada por una medicina positivista, por las dificultades de acceso, la falta de acogida y protección de los sistemas médicos, y por diversos intereses que priman en la medicina administrada. Es debido a estas tensiones que el CI debe seguir siendo garante de una medicina centrada en el paciente no sólo autónomo, sino primordialmente apoyado en sus expectativas de consejo y ayuda al construirse la relación de mutua confianza a lo largo del curso de la enfermedad.

\section{Referencias}

1. León FJ. Ley de derechos y deberes de las personas en la atención de salud. Una mirada bioética. Rev Med Chile 2012; 140: 1490-4. 
2. Faden RR, Beauchamp TL. A History and Theory of Informed Consent. New York: Oxford University Press, 1986.

3. Roach R. Why is informed consent important? J Med Ethics 2014; 40: 435-6.

4. Rendtorff JD, Kemp P. Basic ethical principles in European bioethics and biolaw. Vol I. Copehagen: Center for Ethics and Law, Barcelona Institut Borja e Bioética; 2000.

5. Osman H. History and Development of the Doctrine of Informed Consent. The International Electronic Journal of Health Education 2001; 4:41-47. http://www.iejhe.org (Accedido el 21 de junio de 2016).

6. García Llerena VM. De la bioética a la biojurídica: el principialismo y sus alternativas. Granada: Editoriales Comares; 2012.

7. Parra DS. La obligación de informar al paciente. Cuestiones sobre el derecho a ser informado. Rev Med Chile 2013; 141: 1578-83.

8. Figueroa RG. Consentimiento informado en la nueva ley de derechos de los pacientes. Rev Med Chile 2012; 140: 1347-51.

9. O’Neill O. Autonomy and Trust in Bioethics. Cambridge UK, Cambridge University Press; 2002.

10. O’Neill O. A Question of Trust. Cambridge UK: Cambridge University Press; 2002.

11. Luna, F. Bioethics and vulnerability: A Latin American View. Value Inquiry Books Series. Amsterdam-NY. RODOPI; 2006.

12. Sanz Rubiales A, del Valle Rivero ML, Fernández González M, Ferreira Alonso R. Teoría y práctica del consentimiento informado. Cuadernos de Bioética 2016/1a; 27 : 69-78.

13. Levy N. Forced to be free? Increasing patient autonomy by constraining it. J Med Ethics 2014; 40: 293-300.

14. Caplan, AL. Why autonomy needs help. J Med Ethics 2014; 40: 301-2.

15. O’Neill O. Some limits of informed consent. J Med
Ethics 2003; 29: 4-7.

16. Tealdi JC. Los principios de Georgetown. En Garrafa V, Kottow M, Saada A. (Cordinadores). Estatuto Epistemológico de la Bioética México DF: UNESCO/UNAM; 2005. pp. 35-54.

17. Flanigan J. Three arguments against prescription requirements. J Med Ethics 2012; 38: 579-86.

18. Dickenson D. Risk and Luck in Medical Ethics. Cambridge UK: Polity; 2003.

19. Wierzba S. Consentimiento. En Tealdi JC (coordinador): Diccionario latinoamericano de bioética. Bogotá: UNESCO/ Universidad Nacional de Colombia; 2008: pp 216-7.

20. Rodríguez, JA. Profesionalismo médico: aspectos históricos y religiosos. Rev Med Chile 2006; 174: 381-4.

21. Siqueira JE. Consentimiento en atención clínica. En Tealdi JC (coordinador): Diccionario latinoamericano de bioética. Bogotá: UNESCO/ Universidad Nacional de Colombia; 2008: pp. 221-5.

22. Álvarez-Díaz JA. ¿Bioética latinoamericana o bioétca en Latinoamérica? Rev Latinoam Bioet 2012; 22: 10-27.

23. Rosselot E. Dimensiones del profesionalismo médico. Proyecciones para el siglo XXI. Rev Med Chile 2006; 134: 657-64.

24. Lew D. Toma de decisiones médicas. En Tealdi JC (coordinador): Diccionario latinoamericano de bioética. Bogotá: UNESCO/ Universidad Nacional de Colombia; 2008: pp 445-448.

25. Eyal N. Using informed consent to save trust. J Med Ethics 2014; 40: 437-44.

26. Cuadrado C.

27. Carvallo A. Médicos y profesionalismo. Pacientes e información. Rev Med Chile 2005; 133: 253-8.

28. Cohn S. 'Trust mi doctor, trust my pancreas': trust as an emergent quality of social practice. Philosophy, Ethics and Humanities in Medicine, 2015; 10: 9. DOI 10.1186/13010-015-0029-6 (Accedido el 20 de junio de 2016). 